Neurasthenia: prevalence, disability and health care characteristics in the Australian community

\author{
IAN HICKIE, TR ACEY DAVENPORT, CATHY ISSAKIDIS and GAVIN ANDREWS
}

\author{
Background Neurasthenia imposes a \\ high burden on primary medical health \\ care systems in all societies.
}

\begin{abstract}
Aims To determine the prevalence of ICD-10 neurasthenia and associated comorbidity, disability and health care utilisation.
\end{abstract}

\author{
Method Utilisation of a national sample \\ of Australian households previously \\ surveyed using the Composite \\ International Diagnostic Interview and \\ other measures.
}

\section{Results Prolonged and excessive} fatigue was reported by 1465 people (13.29\% of the sample). Of these, one in nine people meet current ICD-10 criteria for neurasthenia. Comorbidity was associated with affective, anxiety and physical disorders. People with neurasthenia alone $(<0.5 \%$ of the population) were less disabled and used less services than those with comorbid disorders.

\section{Conclusions Fatigue is frequent in the Australian community and is common in people attending general practice. Neurasthenia is disabling and demanding of services largely because of its comorbidity with other mental and physical disorders. Until a remedy for persistent fatigue is provided, doctors should take an active psychological approach to treatment.}

\section{Declaration of interest None.}

Neurasthenia as a diagnostic entity has a long and chequered history in psychiatry (Wessely, 1990; Hickie et al, 1998). Despite changes in diagnostic fashion, aetiological theorising and modes of treatment, people who essentially report mental and physical fatigue, a range of other neuropsychological and mood symptoms (e.g. impaired concentration and short-term memory, irritable mood, non-restorative sleep) as well as other non-specific physical symptoms (e.g. muscle aches and pains, headache, general malaise) continue to impose a high burden on primary medical health care systems in all societies (Üstün \& Sartorius, 1995; Hickie et al, 2001a).

\section{Classification of neurasthenia}

Although the diagnostic concept fell into disrepute in the English-speaking world in the 20th century (being seen more simply as a variant of depression or anxiety), it persisted in most non-English speaking countries. Consequently, the ICD-10 (World Health Organization (WHO), 1992) contains a clear definition of the concept and the World Psychiatric Association (WPA) has promoted a renewed diagnostic and research effort (WPA, 1999). In doing so, the WPA sought to broaden the concept to include cognitive, emotional, somatic, energy and sleep variables that could give the syndrome specific attributes that are clearly different to the symptoms of depressive and anxiety disorders (WPA, 1999).

The ICD-10 diagnostic criteria for research (WHO, 1993) are included in the chapter on 'Neurotic, stress-related and somatoform disorders' in a subsidiary section entitled 'Other neurotic disorders', implying a disorder of uncertain lineage. The diagnostic criteria for neurasthenia can be summarised as follows:

(a) either, persistent and distressing feelings of exhaustion after minor mental effort or persistent and distressing feelings of fatigue after minor physical effort;

(b) accompanied by one or more of the following symptoms: muscular aches or pains; dizziness; tension headache; sleep disturbance; inability to relax; and irritability;

(c) inability to recover through rest, relaxation or enjoyment;

(d) duration exceeds 3 months;

(e) does not occur in the presence of organic mental disorders, affective disorders or panic or generalised anxiety disorder.

Although the WHO thought neurasthenia sufficiently different to other 'Neurotic, stress-related and somatoform disorders' to justify its own category, research groups have tended to be more conservative. Traditionally, most psychiatric epidemiologists highlight the comorbidity with depressive and anxiety disorders (Wessely, 1990) and have rejected the utility of differentiating the concept until social covariates, course or response to treatment distinguish it from (say) dysthymia (Goldberg \& Bridges, 1991). Such disorders have not been included in the major North American epidemiological studies of the past decade (Kessler et al, 1994). A more proactive view, however, has argued for evidence of independence from depression and anxiety at the levels of multivariate modelling of symptom data (Gillespie et al, 1999), genetic vulnerabilities (Hickie et al, 1999a), longitudinal course (Hickie et al, 1999b) and treatment response. Although such studies link neurasthenia more closely with other somatoform disorders, there is evidence that the category can be distinguished from entities such as fibromyalgia, irritable bowel, somatic depression and somatic anxiety (Kirmayer \& Robbins, 1991).

Although the nosological debate is complex, studies in primary care indicate that prolonged fatigue syndromes are common. Prevalence rates for prolonged fatigue (typically greater than 1 month) vary from 18 to $37 \%$ (Pawlikowska $e t$ al, 1994; Hickie et al, 1996, 2001a), whereas the WHO Primary Care Study (Sartorius et al, 1993) found that an average of $5.3 \%$ of general practice patients met criteria for neurasthenia (range 1.1$10.5 \%)$. All studies have emphasised that the syndrome is associated with disability, chronicity, comorbidity and high service utilisation. The Australian National Survey of Mental Health and Wellbeing (Andrews 
et al, 2001) is the first national community based psychiatric survey to include a module specifically designed to identify people who met criteria for ICD-10 neurasthenia. This paper is an account of the key findings, emphasising not only prevalence rates but also patterns of comorbidity, disability and health care utilisation.

\section{METHOD}

\section{Sample}

The national survey was conducted by the Australian Bureau of Statistics under the terms of their Act that guarantees the privacy of respondents. A multi-stage sample of private dwellings in rural and urban Australia was drawn. Each state and territory was stratified and each dwelling within a stratum had an equal and known probability of selection. In all, 13624 private dwellings were initially selected in the survey sample, and one adult member aged 18 years and over randomly selected as the possible respondent. A total of 10641 people participated, a response rate of $78.1 \%$. The age and gender characteristics of the sample were weighted to match the age and gender distribution in the national census.

\section{Assessment}

The whole interview was administered from a laptop computer. The Composite International Diagnostic Interview (CIDI v 2.1; WHO, 1997) was used to determine, using ICD-10 criteria, the presence of seven anxiety disorders, three affective disorders and four substance use disorders in the $\mathbf{1 2}$ months prior to interview.

Neurasthenia was identified using an interview developed by Tacchini et al (1995). All results in this paper are with the exclusion criteria not applied for other mental or physical disorders. Personality disorders were identified using a screening questionnaire (Loranger et al, 1997).

Disability was measured at the beginning of the interview by the SF-12 (Ware et al, 1996) and by the National Comorbidity Survey 'days out of role' questions. Neuroticism was measured using the 12item version of the Eysenck scale (Eysenck et al, 1985). Demographic and service utilisation data were also obtained. The method of the survey has been described previously (Andrews et al, 2001).

\section{Data analysis}

Routine data analysis procedures were used but, as a result of the complex sample design and weighting, specific software was required to estimate standard errors (s.e.). The s.e. of prevalence estimates and confidence intervals around odds ratios (ORs) derived from logistic regression models were estimated using delete-1 jackknife repeated replication in 30 design-based subsamples (Kish \& Frankel, 1974). These calculations used the SUDAAN software package (Shah et al, 1997).

\section{RESULTS}

\section{Diagnosis}

The criteria for neurasthenia are listed above. Criterion A, prolonged and excessive fatigue, was endorsed by 1465 people $(13.2 \%$ of the sample). These responses were probed to ensure that the symptom was clinically significant and not attributed by the respondent to drugs or alcohol, physical illness or injury (601 persons agreed that this was so). Criterion $\mathrm{C}$ requires that the fatigue does not respond to rest and 318 of the 601 participants endorsed this. Criterion D requires that the fatigue lasts 3 months or more, and this was so for 186 participants. Finally we asked about the presence of the Criterion B symptoms, 172 of the 186 met this criterion. Thus, although complaints of fatigue are common, only one in nine people who complain of fatigue meet current ICD-10 criteria for neurasthenia. As distinct from non-specific complaints of fatigue, neurasthenia is not a common mental disorder.

\section{Prevalence}

Data on the weighted prevalence of neurasthenia are displayed in Table 1 , by age and gender, for people meeting criteria in the past month $(1.2 \%)$ and sometimes in the past year $(1.5 \%)$. Only $20 \%$ of people who met criteria during the year were not current cases. The disorder is chronic. The female to male ratio was small (1.4 and $1.2)$ in contrast to what is often believed, and different from the pattern seen in health care facilities. On further examination of the numbers of people in the population who reported fatigue $(13.2 \%)$, significantly more females than males said 'yes' $(14.9 \% \quad v .11 .3 \% ; P<0.05)$. In addition, when respondents were asked whether it was clinically significant (601 said 'yes'), female rates were still higher but the confidence intervals just overlapped $(6.3 \%$ v. $4.4 \% ; P>0.05)$. For all other criteria (B-D) the rates of endorsement were almost identical for males and females. Thus, although more women than men in the population report fatigue the prevalence of neurasthenia is not higher in women. The multivariate associations of demographic variables are shown in Table 2 together with those for people with any 12-month mental disorder. Both sets of disorders decline with age, both are more common among people who are separated, widowed or divorced, both are more common among those with less education and both are more common among those born in Australia. That is, as the socio-demographic characteristics

Table I Weighted prevalence of I2- and I-month ICD-I0 neurasthenia by age and gender

\begin{tabular}{|c|c|c|c|c|c|c|}
\hline \multirow[t]{3}{*}{ Age } & \multicolumn{3}{|c|}{ I2-month } & \multicolumn{3}{|c|}{ I-month } \\
\hline & Males & Females & Persons & Males & Females & Persons \\
\hline & \% (s.e.) & \% (s.e.) & \% (s.e.) & \% (s.e.) & \% (s.e.) & \% (s.e.) \\
\hline $18-24$ & $1.0(1.2)$ & $2.4(0.9)$ & I.7 (0.7) & $1.0(1.2)$ & $1.6(0.7)$ & I. $3(0.7)$ \\
\hline $25-34$ & I.I (0.4) & $1.8(0.4)$ & $\mathrm{I} .5(0.3)$ & $0.7(0.4)$ & $\mathrm{I} .5(0.4)$ & I.I (0.3) \\
\hline $35-44$ & $1.6(0.3)$ & I.7 (0.6) & $1.6(0.3)$ & I.4(0.3) & I.2(0.6) & I.3 (0.3) \\
\hline $45-54$ & I.8(0.5) & $2.8(0.7)$ & $2.3(0.4)$ & $1.7(0.6)$ & $2.1(0.5)$ & $1.9(0.4)$ \\
\hline $55-64$ & $0.8(0.4)$ & I.I (0.5) & I.0 (0.4) & $0.8(0.4)$ & I.I (0.5) & I.0 (0.4) \\
\hline$>65$ & $0.7(0.4)$ & $0.5(0.2)$ & $0.6(0.2)$ & $0.7(0.4)$ & $0.3(0.2)$ & $0.5(0.2)$ \\
\hline Total & I.2(0.3) & I.7 (0.3) & $1.5(0.2)$ & I.I (0.3) & $\mathrm{I} .3(0.2)$ & $1.2(0.2)$ \\
\hline
\end{tabular}


Table 2 Multivariate associations of socio-demographic correlates for 12-month neurasthenia and any 12-month ICD-10 mental disorder

\begin{tabular}{|c|c|c|c|c|}
\hline \multirow[t]{3}{*}{ Correlates' } & \multicolumn{4}{|c|}{ ICD-10 mental disorder } \\
\hline & \multicolumn{2}{|c|}{ 12-month neurasthenia } & \multicolumn{2}{|c|}{ Any 12-month mental disorder } \\
\hline & Odds ratio & $95 \% \mathrm{Cl}$ & Odds ratio & $95 \% \mathrm{Cl}$ \\
\hline \multicolumn{5}{|l|}{ Age } \\
\hline $18-24$ & 1.0 & - & 1.0 & - \\
\hline $25-34$ & 1.0 & $0.4-2.9$ & 1.0 & $0.8-1.3$ \\
\hline $35-44$ & I.I & $0.3-4.3$ & 1.0 & $0.8-1.2$ \\
\hline $45-54$ & 1.6 & $0.5-4.8$ & 0.8 & $0.6-1.1$ \\
\hline $55-64$ & $0.4^{*}$ & $0.2-1.0$ & $0.4^{* *}$ & $0.3-0.6$ \\
\hline$>65$ & 0.4 & $0.1-1.8$ & $0.2^{* *}$ & $0.1-0.2$ \\
\hline$\chi_{5}^{2}(P)$ & 17.7 & 0.003 & 218.5 & $<0.001$ \\
\hline \multicolumn{5}{|l|}{ Marital status } \\
\hline Married/de facto & 1.0 & - & 1.0 & - \\
\hline Separated/divorced/widowed & $2.4^{*}$ & $1.5-3.7$ & $2.0^{* *}$ & $1.5-2.5$ \\
\hline Never married & 1.2 & $0.6-2.3$ & $1.5^{* *}$ & $1.3-1.8$ \\
\hline$\chi_{2}^{2}(P)$ & 14.4 & $0.00 \mathrm{I}$ & 48.9 & $<0.001$ \\
\hline \multicolumn{5}{|l|}{ Education } \\
\hline Bachelor degree or higher & 1.0 & - & 1.0 & - \\
\hline Diploma & 0.8 & $0.2-4.2$ & 1.2 & $0.9-1.6$ \\
\hline Vocational qualification & 1.9 & $0.6-6.2$ & $1.5^{*}$ & $1.1-2.0$ \\
\hline High school only & 2.3 & $0.6-8.1$ & $1.6 * *$ & $1.3-1.8$ \\
\hline$\chi_{3}^{2}(P)$ & 7.9 & 0.048 & 36.3 & $<0.001$ \\
\hline \multicolumn{5}{|l|}{ Employment } \\
\hline Employed $(\mathrm{f} / \mathrm{t}$ or $\mathrm{p} / \mathrm{t})$ & - & - & 1.0 & - \\
\hline Short-term unemployed ${ }^{3}$ & - & - & $1.6 *$ & $1.1-2.3$ \\
\hline Long-term unemployed ${ }^{4}$ & - & - & $2.6^{* *}$ & $1.8-3.8$ \\
\hline Not in the labour force & - & - & $1.6^{* *}$ & $1.3-1.9$ \\
\hline$\chi_{3}^{2}(P)$ & - & NS & 38.5 & $<0.001$ \\
\hline \multicolumn{5}{|l|}{ Country of birth } \\
\hline Australia & 1.0 & - & 1.0 & - \\
\hline Other English speaking country & 0.5 & $0.2-1.0$ & 0.9 & $0.7-1.2$ \\
\hline Other non-English speaking country & 0.6 & $0.3-1.1$ & $0.8^{*}$ & $0.6-1.0$ \\
\hline$\chi_{2}^{2}(P)$ & 6.6 & 0.037 & 6.6 & 0.037 \\
\hline
\end{tabular}

$\mathrm{f} / \mathrm{t}$, full-time; $p / t$, part-time; NS, non-significant.

$* P<0.05, * * P<0.001$.

I. Gender, urban v. rural residence, employment status and language used at home were not significant in unadjusted models and were therefore not included in the multivariate model.

2. Source: Andrews et al (200I)

3. Unemployed $<12$ months.

4. Unemployed $\geqslant 12$ months.

of neurasthenia are similar to other mental disorders, it is likely that social risk factors are shared. Employment is not significant in neurasthenia, whereas psychological morbidity generally is associated with not being in the labour force.

Comorbidity is regarded as a hallmark of neurasthenia. In Table 3 we present data on the prevalence of comorbid disorders among people with neurasthenia. In Model 1 we show that there is more comorbidity with major depression, panic disorder and generalised anxiety disorder than could be expected by chance after adjustment for the prevalence of the comorbid disorder and the average level of comorbidity of that disorder. These are the disorders specified as exclusion criteria in ICD-10. In Model 2 we calculate the same information, not for individual disorders but for disorder groups. Now the significant associations are with affective, anxiety and personality disorders. Naturally the association with any mental disorder is significant, as is the association with any self-reported physical disorder. People who meet criteria for neurasthenia report symptoms that suggest they are at increased risk for specific mental and any physical disorder. Their risk of a substance misuse disorder is not increased.

When comorbidity is endemic it is difficult to know whether the attribute being measured belongs to the target disorder or to the comorbid disorder. In clinical practice, when the patient has more than one disorder, the patient and doctor agree on a priority and usually deal with the main problem first. In the survey, after all disorders had been enumerated, we listed the groups of symptoms they had complained of, and asked people who had met criteria for more than one disorder: 'Which of these problems troubles you the most?'. We regarded this as the patient's main problem. In 13 people neurasthenia was the only disorder present, whereas a further 36 who did have comorbid disorders, identified neurasthenia as their main problem. Thus, neurasthenia was the main problem in 49 people (less than $0.5 \%$ of the population). Of the remainder of people with neurasthenia and comorbid disorders, 50 nominated an affective disorder as their main problem, 39 an anxiety disorder and 31 a physical disorder as their main problem. Three people thought personality or substance misuse disorders were their main problem.

In Table 4 we present data on neuroticism, disability measured by the SF-12 and by disability days, and service utilisation in terms of consultations and hospital admissions. People with neurasthenia as a main problem were less likely to be comorbid with a mental or a physical disorder, less disabled and used fewer services than the complete group. We then examined all people with neurasthenia, divided into those with neurasthenia as a main problem and those with neurasthenia who identified affective, anxiety or physical disorders as their main problem. People with neurasthenia as a main problem were less disabled $(P=0.026)$ and used fewer services $(P=0.005)$ than did the other three groups.

\section{DISCUSSION}

Complaints of fatigue are frequent in the general population and are particularly common in people attending general 
Table 3 Weighted prevalence and odds ratios of comorbid ICD-10 mental disorders and any physical disorder among persons with 12-month neurasthenia

\begin{tabular}{|c|c|c|}
\hline \multirow[t]{2}{*}{ Comorbid disorder' } & \multicolumn{2}{|c|}{$\begin{array}{c}\text { Prevalence of disorder among persons with 12-month } \\
\text { neurasthenia }\end{array}$} \\
\hline & \% (s.e.) & $\mathrm{OR}^{\prime}(95 \% \mathrm{Cl})$ \\
\hline \multicolumn{3}{|l|}{ Model I: Individual disorders } \\
\hline Major depression & $50.4(4.6)^{* *}$ & $5.2(3.0-9.0)$ \\
\hline Dysthymia & $14.6(2.7)$ & $1.5(0.7-3.2)$ \\
\hline Panic with or without agoraphobia & $27.8(3.9)^{*}$ & $2.6(1.4-4.9)$ \\
\hline Social phobia & $19.0(4.8)$ & $2.2(0.8-6.3)$ \\
\hline Generalised anxiety disorder & $40.4(4.6)^{*}$ & $2.7(1.3-5.4)$ \\
\hline Obsessive-compulsive disorder & $3.1(1.9)$ & $0.7(0.2-2.2)$ \\
\hline Post-traumatic stress disorder & $27.5(8.5)$ & $2.7(0.8-9.5)$ \\
\hline Alcohol misuse or dependence & $14.0(3.3)$ & $0.8(0.4-1.6)$ \\
\hline Drug misuse or dependence & $10.0(2.4)$ & $2.0(0.9-4.6)$ \\
\hline \multicolumn{3}{|l|}{ Model 2: Disorder groups } \\
\hline Any affective disorder & $53.7(4.4)^{* *}$ & $5.2(3.3-8.1)$ \\
\hline Any anxiety disorder & $64.9(4.3)^{* *}$ & $6.6(4.2-10.6)$ \\
\hline Any substance use disorder & $19.9(4.1)$ & I.I (0.5-2.I) \\
\hline Any personality disorder & $35.5(4.7)^{*}$ & $2.0(1.2-3.5)$ \\
\hline Model 3: Any other mental disorders & $79.3(4.8)^{* *}$ & $14.4(7.9-26.5)$ \\
\hline Model 4: Any physical disorder ${ }^{2}$ & $62.3(4.4)^{* *}$ & $2.7(1.8-4.0)$ \\
\hline \multicolumn{3}{|c|}{$\begin{array}{l}* P<0.05 ; * * P<0.001 \text {. } \\
\text { I. ORs were calculated using parameter estimates from logistic regression models and they represent the odds of hav- } \\
\text { ing each comorbid disorder (single or group) for persons with neurasthenia compared to persons without neurasthe- } \\
\text { nia. The ORs were derived from four different models: Model I - each single mental disorder controlling for the } \\
\text { presence of all other single mental disorders; Model } 2 \text { - each disorder group controlling for the presence of all other } \\
\text { disorder groups; Model } 3 \text { - containing one dichotomous variable (any other mental disorder v. no other mental disor- } \\
\text { der); and Model } 4 \text { - containing one dichotomous variable (any physical disorder v. no physical disorder). } \\
\text { 2. Any physical disorder was defined as presence of at least one of } I 2 \text { conditions: asthma, chronic bronchitis, anaemia, } \\
\text { high blood pressure, heart trouble, arthritis, kidney disease, diabetes, cancer, stomach ulcer, chronic liver trouble or } \\
\text { hernia rupture. }\end{array}$} \\
\hline
\end{tabular}

practitioners. Generally, the rates of most mental disorder in primary care patients are increased over community rates by a factor of 2-3 (Hickie et al, 2001b). In this national community survey, $13.2 \%$ of the Australian adult population report prolonged and excessive fatigue as a problem. Previous Australian general practice surveys indicate prevalence rates of $25-37 \%$ (Hickie et al, 1996, 2001a). This study indicates that the prevalence of the more chronic and disabling syndrome of neurasthenia is $1.5 \%$ in the general population and this is consistent with Australian and international studies in primary care that report rates of neurasthenia $(1.3-5.2 \%$; Sartorius et al, 1993; Hickie et al, 1996). There have been a series of community and primary care-based studies for the closely related condition of chronic fatigue syndrome $(6$ months of prolonged and excessive fatigue without other medical or psychiatric cause). Here community estimates range from 0.2 to $0.7 \%$ (Buchwald et al, 1995; Lawrie et al, 1997; Jason et al, 1999) and primary care estimates from 0.5 to $2.5 \%$ (Bates et al, 1993; Wessely et al, 1997).

Although neurasthenia is by definition prolonged ( $>3$ months), this study indicates that it is chronic, with $80 \%$ of people who met criteria in the past 12 months also being current cases. This is consistent with our previous longitudinal

Table 4 Chronicity, neuroticism, disability and health service utilisation among all persons with 12-month neurasthenia, those with neurasthenia as their principal complaint and among those with an anxiety, affective or a physical disorder as their principal complaint

\begin{tabular}{|c|c|c|c|c|c|}
\hline & \multicolumn{5}{|c|}{ I2-month neurasthenia' } \\
\hline & $\begin{array}{c}\text { All I2-month } \\
\text { neurasthenia }(n=\mid 72)\end{array}$ & $\begin{array}{c}\text { A. Neurasthenia as } \\
\text { main problem }(n=49)\end{array}$ & $\begin{array}{l}\text { B. Affective as main } \\
\text { problem }(n=50)\end{array}$ & $\begin{array}{l}\text { C. Anxiety as main } \\
\text { problem }(n=39)\end{array}$ & $\begin{array}{l}\text { D. Physical illness as } \\
\text { main problem }(n=3 I)\end{array}$ \\
\hline \multicolumn{6}{|l|}{ Neuroticism, mean (s.e.) } \\
\hline EPQ-N & $6.7(0.3)$ & $5.7(0.5)$ & $7.4(0.8)$ & $8.3(0.9)$ & $5.7(0.6)$ \\
\hline \multicolumn{6}{|l|}{ Disability, mean (s.e.) } \\
\hline SF-12 mental component score & $36.4(I . I)$ & $42.5(1.5)$ & $31.4(2.7)^{*}$ & $34.3(2.5)^{*}$ & $36.6(4.5)$ \\
\hline SF-12 physical component score & $40.4(1.0)$ & 44.7 (I.9) & $42.3(2.3)$ & $37.6(2.3)$ & $33.7(2.8)$ \\
\hline Disability days & $12.8(I .1)$ & $7.5(2.4)$ & $16.2(2.5)$ & $15.3(2.2)$ & $13.1 \quad(3.0)$ \\
\hline \multicolumn{6}{|l|}{ Service utilisation, \% (s.e.) } \\
\hline Any consultation ${ }^{2}$ & $95.8(2.1)$ & $87.2(8.0)$ & $100(-)$ & $97.5(4.0)$ & $100(-)$ \\
\hline Any mental health consultation ${ }^{3}$ & $60.5(4.6)$ & $50.9(11.9)$ & $88.4(4.8)^{*}$ & $61.2(10.8)$ & 38.1 (II.7) \\
\hline Any hospital admission ${ }^{4}$ & $23.0(3.1)$ & $9.2(5.6)$ & $27.3(7.2)$ & $17.8(8.2)$ & $46.6(14.6)^{*}$ \\
\hline
\end{tabular}

$* P<0.05$, for comparison with A. Neurasthenia as main problem.

I. There were three people with personality or substance misuse disorders as their main complaint. Groups A-D were compared on disability and health service utilisation variables using polytomous logistic regression with a four level dependent variable, coded according to main problem diagnosis (neurasthenia as main problem, v. affective disorder as main problem, anxiety disorder as main problem, physical disorder as main problem).

2 . Refers to any consultation in the previous 12 months with any health professional for any reason.

3. Refers to any consultation in the previous 12 months with any health professional for mental health problems.

4. Refers to any hospital admission for any reason in the previous 12 months. 
reports in primary care that emphasised early ages of onset and chronic course (Hickie et al, 1999b). The multivariate associations between age, gender, marital status, education and country of birth are also similar to those identified for other mental disorders in the wider survey. People who meet criteria for neurasthenia do report more symptoms of anxiety, affective and physical disorders than is expected, even after allowing for the probability of association and level of comorbidity in the other disorders. Both these patterns are consistent with the notion that neurasthenia is indeed, typically, a mental disorder. The patterns of comorbidity are very similar to patients with neurasthenia seen in clinical settings (Farmer et al, 1995).

Interestingly, neuroticism levels were not increased in persons with neurasthenia as their main problem. This is consistent with other reports (Chubb et al, 1999). This could suggest that such people are different not only at a symptom-reporting level (emphasising more overtly physical rather than psychological symptoms) but also at the level of important vulnerability factors. Given the evidence from other genetic modelling studies, it could be seen as consistent with less relevance for traditional psychological risk factors in this patient group (Farmer et al, 1999; Hickie et al, 1999a).

When people with neurasthenia were subdivided according to the disorder that they regarded as their main problem, people with neurasthenia as a main problem were less disabled and used fewer services than the others with neurasthenia who regarded other disorders as their main problem. This suggests that the degree of disability and service use typically associated with neurasthenia is more because of the comorbid symptoms (depression and anxiety) than prolonged fatigue. In this survey people with neurasthenia as a main problem did not differ in these respects from the large numbers of people who identified, irrespective of neurasthenia, depression or anxiety as their main problem (see Andrews et al, 2001). We argue that neurasthenia is recognised as disabling and demanding of services largely because of its comorbidity with other affective, anxiety and physical disorders. However, as with other disorders, the higher the degree of 'comorbidity' the higher the rates of disability and health care service utilisation.

\section{CLINICAL IMPLICATIONS}

While complaints of prolonged and excessive fatigue are common in the community, the syndrome of neurasthenia is uncommon.

- Neurasthenia is typically chronic and is associated with high levels of comorbid affective and anxiety disorders.

More pure forms of neurasthenia are associated with lower levels of neuroticism, disability and health care utilisation.

\section{LIMITATIONS}

Cross-sectional surveys provide only limited insight into the nature of the association between neurasthenic and affective and anxiety disorders.

- The clinical significance of the disorders identified by such community surveys is inferred from self-reported disability data.

- Self-reported comorbid physical disorders are assumed to reflect clinically significant medical conditions.

AN HICKIE, FRANZCP, School of Psychiatry, University of New South Wales, Sydney, Australia; and Chief Executive Officer, 'beyondblue: the national depression initiative', Melbourne, Australia; TRACEY DAVENPORT, BA (Hons), School of Psychiatry, University of New South Wales at St George Hospital, Sydney, Australia; CATHY ISSAKIDIS, BA (Hons),World Health Organization Collaborating Centre for Evidence for Mental Health Policy; and, School of Psychiatry, University of New South Wales at St Vincent's Hospital, Sydney, Australia; GAVIN ANDREWS, FRCPsych, World Health Organization Collaborating Centre for Evidence for Mental Health Policy; and, School of Psychiatry, University of New South Wales at St Vincent's Hospital, Sydney, Australia

Correspondence: Professor lan Hickie, Academic Department of Psychiatry, 7 Chapel Street, Kogarah, NSW 2217, Australia. Tel: +6I 29350 2035; fax: +6I 29350 2098; e-mail: ian. hickie@beyondblue.org.au

(First received 17 January 2002, accepted 21 March 2002)

Such cross-sectional surveys can provide only limited insights. Other longitudinal work (Hickie et al, 1999b; Addington et al, 2001) has emphasised that when fatigue and psychological symptoms co-occur, persons are at high risk of going on to experience further episodes again characterised by both prolonged fatigue and psychological disorder. Those experiencing prolonged fatigue only, however, appear to have a more stable pattern of future fatigue without increased rates of later psychological disorder. Along with other genetic and treatment data, this suggests differing aetiological and illness course determinants. All such studies imply that when prolonged fatigue occurs in the context of other dysphoria that doctors should take an active psychological approach to treatment. It is likely, however, that medical and psychological debate surrounding prolonged fatigue states will persist until doctors provide a remedy for persistent idiopathic fatigue.

\section{ACKNOWLEDGEMENTS}

This paper was supported by a contract from the Australian Department of Health and Aged Services to the WHO Collaborating Centre for Evidence in Mental Health Policy, Sydney, to support a survey data analysis consortium (G.A., V. Carr, G. Carter, R. Crino,W. Hall, A. Henderson, I.H., C. Hunt, L. Lampe, J. McGrath, A. McFarlane, P. Mitchell, L. Peters, M. Teesson and K.Wilhelm). The survey was conducted by the Australian Bureau of Statistics, who do not necessarily endorse the view expressed in this paper.

\section{REFERENCES}

Addington, A. M., Gallo, J. J., Ford, D. E., et a (200I) Epidemiology of unexplained fatigue and major depression in the community: the Baltimore ECA 
follow-up, 1981-1994. Psychological Medicine, 31, 1037-1044.

\section{Andrews, G., Henderson, S. \& Hall, W. (200I)}

Prevalence, comorbidity, disability and service utilisation: overview of the Australian National Mental Health Survey. British Journal of Psychiatry, 178, 145-153.

\section{Bates, D. W., Schmitt, W., Buchwald, D., et al (1993)} Prevalence of fatigue and chronic fatigue syndrome in a primary care practice. Archives of Internal Medicine, 153, 2759-2765.

Buchwald, D., Umali, P., Umali, J., et al (1995) Chronic fatigue and the chronic fatigue syndrome: prevalence in a Pacific Northwest health care system. Annals of Internal Medicine, 123, 81-88.

Chubb, H. L., Jones, I., Hillier, J., et al (1999) Chronic fatigue syndrome - personality and attributional style of patients in comparison to healthy controls and depressed individuals. Journal of Mental Health, $\mathbf{8}$, $351-359$.

Eysenck, S. B. G., Eysenck, H. J. \& Barratt, P. A. (1985) A revised version of the psychoticism scale. Personality and Individual Differences, 6, 21-29.

Farmer, A., Jones, I., Hillier, J., et al (1995) Neurasthenia revisited: ICD-10 and DSM-III-R psychiatric syndromes in chronic fatigue patients and comparison subjects. British Journal of Psychiatry, $\mathbf{1 6 7}$ 503-506.

_, Scourfield, J., Martin, N., et al (1999) Is disabling fatigue in childhood influenced by genes? Psychological Medicine, 29, 279-282.

Gillespie, N., Kirk, K. M., Heath, A. C., et al (1999) Somatic distress as a distinct psychological dimension. Social Psychiatry and Psychiatric Epidemiology, 34, $451-458$.

Goldberg, D. P. \& Bridges, K.W. (199I) Minor psychiatric disorders and neurasthenia in general practice. In Problems of Psychiatry in General Practice (eds M. Gastpar \& P. Kielholz), pp. 79-88. Lewiston, NY: Hogrefe \& Huber Publishers.

Hickie, I., Kirk, K. \& Martin, N. (1999a) Unique genetic and environmental determinants of prolonged fatigue: a twin study. Psychological Medicine, 29. 259-268.
_ , Koschera, A., Hadzi-Pavlovic, D., et al (1999b) The temporal stability and co-morbidity of prolonged fatigue: a longitudinal study in primary care. Psychological Medicine, 29, 855-861.

Hickie, I. B., Hooker, A. W., Hadzi-Pavlovic, D., et al (1996) Fatigue in selected primary care settings: sociodemographic and psychiatric correlates. Medical Journal of Australia, 164, 585-588.

_, Scott, E. M. \& Davenport, T. A. (1998) Somatic distress: developing more integrated concepts. Current Opinion in Psychiatry, II, 153-158.

_ , Davenport, T. A., Naismith, S. L., et al (200la) Conclusions about the assessment and management of common mental disorders in Australian general practice. Medical Journal of Australia, 175 (Suppl), S52-S55.

\section{Hadzi-Pavlovic, D., et al (200Ib)}

Development of a simple screening tool for common mental disorders in general practice. Medical Journal of Australia, 175 (Suppl), SIO-SI7.

Jason, L. A., Richman, J. A., Rademaker, A. W., et al (1999) A community-based study of chronic fatigue syndrome. Archives of Internal Medicine, 159, 2129-2137.

Kessler, R. C., McGonagle, K. A., Zhao, S., et al (1994) Lifetime and I2-month prevalence of DSM-III-R psychiatric disorders in the United States. Results from the National Comorbidity Survey. Archives of General Psychiatry, 51, 8-19.

Kirmayer, L. J. \& Robbins, J. M. (1991) Functiona somatic syndromes. In Current Concepts of Somatization Research and Clinical Perspectives (eds L. J. Kirmayer \& J. M. Robbins), pp. 79-106. Washington, DC: American Psychiatric Press, Inc

Kish, L. \& Frankel, M. R. (1974) Inference from complex samples. Journal of the Royal Statistical Society (Series B), 36, I-37.

Lawrie, S. M., Manders, D. N., Geddes, J. R., et a (1997) A population-based incidence study of chronic fatigue. Psychological Medicine, 27, 343-353.

Loranger, A.W., Janca, A. \& Sartorius, N. (1997) Assessment and Diagnosis of Personality Disorders. Cambridge: Cambridge University Press.
Pawlikowska, T., Chalder, T., Hirsch, S. R., et al (1994) Population based study of fatigue and psychological distress. BMJ, 308, 763-766.

Sartorius, N., Üstün, T. B., Costa e Silva, J. A., et al (1993) An international study of psychological problems in primary care. Preliminary report from the World Health Organization Collaborative Project on 'Psychological Problems in General Health Care'. Archives of General Psychiatry, 50, 819-824.

Shah, B.V., Barnwell, B. G. \& Biegler, G. S. (1997) SUDAAN User's Manual. Research Triangle Park, NC: Research Triangle Institute.

Tacchini, R., Janca, A. \& Issacs, M. (1995)

Neurasthenia. Geneva: Division of Mental Health, World Health Organization.

Üstün, T. B. \& Sartorius, N. (1995) Mental lliness in General Health Care: An International Study. Chichester: John Wiley and Sons.

Ware, J. E., Kosinski, M. \& Keller, S. D. (1996) A 12 item short-form health survey: construction of scales and preliminary tests of reliability and validity. Medical Care, 34, 220-233.

Wessely, S. (1990) Old wine in new bottles: neurasthenia and "ME". Psychological Medicine, 20, 35-53.

_ Chalder, T., Hirsch, S., et al (1997) The prevalence and morbidity of chronic fatigue and chronic fatigue syndrome: a prospective primary care study. American Journal of Public Health, 87, 1449-1455.

World Health Organization (1992) International Classification of Disease and Related Disorders (IDC-10). Geneva: World Health Organization.

- (1993) The ICD-10 Classification of Mental and Behavioural Disorders: Diagnostic Criteria for Research. Geneva: World Health Organization.

_ (1997) Composite International Diagnostic Interview - Version 2.I. Geneva: World Health Organization.

World Psychiatric Association (1999) World Psychiatric Association Consensus Statement on the Syndrome of Neurasthenia. Chatswood, NSW: Excerpta Medica Communications. 